I.ES CORRÉLATIONS PHÉNOTYPIQUES ENTRE QUELQUES INDEX DE CONSTITUTION, QUELQUES MESURES DU CORPS ET QUELQUES CARACTËRES D'UTIIISATION APRÉS L'ABATTAGE DES AGNEAUX MERINOS POLONAIS AGÉS DE QUATRE MOIS

\author{
Krystyna ZATUSKA, Z. BERNACKI, K. HELLER \\ Académie Technique et Agronomique Bydgoszcz Pologne
}

Des essais expérimentaux effectués sur 50 agneaux de la race Mérinos polonais ont démontré que chez des agneaux mâles il y a une haute corrélation phénotypique, significative entre des valeurs de quelques index de constitution et quelques mesures du corps (largeur de la poitrine et largeur de la croupe dans les joints de hanches) effectuées avant l'abattage et entre le poids de viande, graisse et d'os dans la demi-carcasse.

Pour les agneaux femelles on a obtenu des coefficients élevés et significatifs de corrélations phénotypiques entre la largeur de la poitrine et entre le poids de viande, et d'os dans la carcasse. Le pourtour de la cuisse mesuré avant l'abattage était en corrélation haute et significative avec le poids de viande et d'os dans la demi-carcasse chez les agneaux de deux sexes.

OPTIMALE RESTRIKTIONEN VON LEISTUNGSMERKMALEN

UNTER GLEICHZEITIGER VERWENDUNG MEHRERER SELEKTIONSINDICES IN EINER POPULATION

\title{
E. NIEBEI
}

Institut f. Tierhaltung u. Tierzüchtung d. Universität Hohenheim, D jooo Stuttgart jo (B.R.D.)

Aus mehreren Arbeiten sind bislang Methoden bekannt, mit denen der Zuchtfortschritt maximiert werden kann unter der Restriktion einer vorgegebenen Anderung weiterer Leistungseigenschaften. Die Anwendung dieser Methoden ist in der Tierzüchtung nur dann optimal, wenn für alle Tiere, die am Aufbau der Folgegeneration beteiligt sind, der gleiche Selektionsindex verwendet wird. Liegen in einer Population jedoch Tiergruppen mit unterschiedlichen Selektionsindices vor, dann sind unter restriktiven Bedingungen die Gewichte der phänotypischen Merkmalswerte eines einzelnen Indexes in Abhängigkeit von den Gewichten sämtlicher Selektionsindices und dem Genanteil sowie der Selektionsschärfe der beteiligten Tiergruppen zu ermitteln. Dafür wurden zwei iterative Rechentnethoden, das NEWTON'sche Näherungsverfahren und ein Gesamtgleichungssystem angewandt bzw. entwickelt. Mit diesen Methoden kann die auferlegte Restriktionsanforderung erfüllt und gleichzeitig der Selektionserfolg der Population maximiert werden.

\section{GENETISCHE ASPEKTE DES LACTOFERRINS IN DER KUHMILCH}

\section{B. SENFT, F. MEYER, R. RUDOLPHI}

Institut jür Tierzucht u. Haustiergenetik, Abt. Biochem. Genetik, Universität Giessen, Bismarckstr. I6, D 6300 Lahn-Giessen, B.R.D.

Die bisherigen Bemiihungen zur Bekämpfung von Mastitis haben nicht zu den erwarteten Erfolgen geführt. Daher wird in den letzten Jahren vermehrt versucht, das natürliche Abwehrsystem des Euters durch Züchtung zu verbessern und somit die Häufigkeit von Sekretionsstörungen zu verringern. Voraussetzung dafür sind Kenntnisse über die körpereigenen Abwehrmechanismen, insbesondere über die Wirkung spezifischer Proteine auf euterpathogene Erreger. Dem eisenbindenden Protein Lactoferrin kommt hierbei eine vorrangige Bedeutung zu. In vitro konnte eine bakteriostatische Wirkung des Lactoferrins gegenüber euterpathogenen Erregern nachgewiesen werden.

Für die vorliegenden Untersuchungen standen Milchproben von I $178 \mathrm{Kühen} \mathrm{zur} \mathrm{Verfügung.}$

Zwischen der Konzentration der Milch an Lactoferrin und dem Gehalt an Laktose ergab sich ein signifikanter Korrelationskoeffizient von $r=-0,50$. Für die Schätzung des erblich bedingten Anteils an der Gesamtvarianz der Lactoferrinkonzentration dienten insgesamt 55 Nachkommengruppen mit durchschnittlich 9,4 Töchtern. Der errechnete Wert betrug $h^{2}=0,15$. Zwischen den 
Nachkommengruppen bestanden bei gleichen Laktationsstadien signifikante Unterschiede zwischen den Mittelwerten für die Konzentration der Milch an Lactoferrin; der höchste Wert betrug $772 \mathrm{mcg} / \mathrm{ml}$ Molke, der niedrigste $34 \mathrm{mcg} / \mathrm{ml}$.

\author{
ESTIMATION OF PARAMETERS FOR CROSSBREEDINGS \\ BETWEEN US BROWN SWISS AND BAVARIAN BRAUNVIEH CATTLE
}

\title{
A. CLEMENT
}

Lehrstuhl für Tierzucht der Technischen Universilät München, D 8050 Freising-Weihenstephan, B.R.D.

Parameters for crossbreedings between Bavarian Braunvieh and Brown-Swiss cattle are estimated. Genetical and environmental aspects of the model, as well as problems concerning the estimation are discussed in this work. Although results may be disputable, strong evidence seems to point out that additive-genetic difference between breeds should be used to increase milking performance. Heterosis-effects are found to be of minor importance. Although the generation-by-generation coadaptation of integrating gene-pools is assumed to be negligible, the estimation of recombination-loss still represents a problem. Standard-deviations of the least-squares estimates of cross-breeding-parameters are very high. Nevertheless, the use of estimable functions permits a prediction of yields according to the Brown-Swiss proportion of genes in cows.

EINFIUSS VON RASSE, SCHIACHTGEWICHT UND FU'TTERNIVEAU
AUF SPEZIELIF FIEISCHLEISTUNGSEIGENSCHAFTHN BEIM RIND

A. EI, HAKIM, H. EICHINGER und F. PIRCHNER

Lehrstuhl für Tierzucht der Technischen Universität München, D 8050 Freising-Weihenstephan, B.R.D.

Die varianzanalytische Auswertung des Versuches zeigt deutliche Rassen- und Gewichtsgruppenunterschiede bei den Fleischbeschaffenheitseigenschaften, wohingegen das Fütterungsniveau aufgrund der bisher vorliegenden Ergebnisse keine statistisch signifikanten Einflusse verursacht. Die Fleckviehrasse weicht bei sensorischen Eigenschaften etwas ab, evtl. ausmastbedingt, während die 3 weiteren Rassen auf ziemlich einheitlichem Niveau liegen. Die gestaffelte Schlachtung nach definierten Gewichtsgruppen führte zu der bekannten mit dem Alter verbundenen Verringerung der hauptsächlichen sensorischen Eigenschaften. Für die einzelnen Rassen ergaben sich hier jedoch deutliche Interaktionen, welche ggf. für die Produktionstechnik von Interesse sein dürften.

Bekanntlich sind Aussagen für sensorische Eigenschaften von Fleisch auch bei Standardisierung der Zubereitung und einer ausgewählten Prüfergruppe mit größeren Streuungen behaftet als meßtechnisch erfassbare Kriterien. Insofern ist das bisher vorliegende Tiermaterial für definitive Aussagen sicher zu klein. Eine Wiederholung des vorgenannten Versuches wird aber bereits durchgeführt.

SELECTION EFFICIENCY WHEN USING A SELECTION INDEX INCLUDING AN ALL-OR-NONE TRAIT AND A NORMALIY DISTRIBUTED TRAIT

O. DANNELL and K. RONNINGEN

Department of Animal Breeding, Agricultural College, S-750o7 Uppsala 7, Sweden

All-or-none traits are often included in selection indexes but the properties of such indexes do not seem to have been investigated exhaustively. This study is an attempt to examine the efficiency of a selection index for mass selection - including an all-or-none trait, together with a continuous one - by means of actual response at different selection intensities. Data have been generated for 180 combinations of parameters for the two traits. The results indicate that in many practical situations where important binomial traits are included in the index, the responses obtained may turn out to be considerably lower than expected from index theory. 